\title{
Colorimetria da madeira de trinta espécies tropicais
}

\author{
Rildo Alexandre Fernandes da Silva ${ }^{\star}$, Carine Setter, Samara Silva Mazette, Rafael Rodolfo de Melo, \\ Diego Martins Stangerlin
}

Instituto de Ciências Agrárias e Ambientais, Universidade Federal de Mato Grosso - Campus de Sinop, Sinop, MT, Brasil.

\begin{abstract}
RESUMO A cor como método empírico de identificação é subjetiva, no entanto pode-se utilizar o método de mensuração quantitativa $\mathrm{CIEL} L^{*} a^{*} b^{*}$. O presente trabalho tem por objetivo realizar a caracterização colorimétrica da madeira do cerne de trinta espécies tropicais. Para tanto foram medidos os parâmetros colorimétricos $\left(\mathrm{L}^{\star}, \mathrm{a}^{\star}, \mathrm{b}^{\star}, \mathrm{C}\right.$ e $\left.\mathrm{h}^{\star}\right)$, mediante o emprego de um colorímetro, e realizada a determinação da massa específica aparente. Os resultados foram tratados em teste de Skott-Knott (95\% de significância) com posterior análise multivariada de Cluster. A análise de clusters dividiu os materiais em cinco grupos, em que nos primeiros estiveram as madeiras mais escuras e pesadas (e.g. Dialium guianese e Caesalpinia echinata), enquanto nos últimos estiveram as madeiras mais claras e leves (Ochroma pyramidale, Simarouba amara e Schizolobium amazonicum). Palavras-chave: cor da madeira; qualidade da madeira; Clusters; propriedades físicas; $\mathrm{CIE} L^{*} a^{*} b$.
\end{abstract}

\section{Colorimetry of wood from thirty tropical species}

\begin{abstract}
The color is a subjective parameter for wood identification, however with the CIE $L^{\star} a^{\star} b$ quantitative measurement it is possible to transform in an objective method. This study aims to characterize the color of heartwood from thirty tropical species. The colorimetric parameters $\left(\mathrm{L}^{\star}, \mathrm{a}^{\star}, \mathrm{b}^{\star}, \mathrm{C}\right.$ and $\left.\mathrm{h}{ }^{\star}\right)$ were measured using a colorimeter. In addition, the wood density was determined for all woods. The results were analyzed by the Scott-Knott test (95\% significance level) and multivariate analysis by the clustering method. The Cluster analysis divided the woods in five groups. One of the groups contained dark and dense woods (e.g. Dialium guianese e Caesalpinia echinata), while other group attached light and less dense woods (Ochroma pyramidale, Simarouba amara e Schizolobium amazonicum ).
\end{abstract}

Keywords: Wood colour; wood quality; Clusters; physical properties; CIE $L^{\star} a^{\star} b$.

\section{Introdução}

Nos últimos anos, o aumento da demanda por madeira tem ampliado a variedade de espécies nativas comercializadas, assim como o emprego dessas em bens de alto valor comercial. Um dos pontos de atratividade sobre a madeira nativa na movelaria são seus atributos estéticos, dados por algumas de suas propriedades organolépticas (propriedades sensoriais), tais como: cor, brilho, desenho, etc.

A cor pode ser um meio de valorização de um produto madeireiro, através de sua comparação com madeiras apreciadas no mercado, tais como; cerejeira, mogno e sucupira, entre outras (CAMARGO; GONÇALEZ, 2001).
Segundo Mori et al. (2004), a coloração da madeira ocorre em uma amplitude colorimétrica desde bege claro até marrom escuro (quase preto), passando por tonalidades mais vibrantes como em madeiras amarelas, avermelhadas, roxas ou alaranjadas. A diferença de tonalidades ocorre entre espécies, como também pode estar dentro de uma mesma espécie e/ou um mesmo indivíduo arbóreo, sendo influenciada por fatores genéticos e ambientais. Gonçalez (1993) descreveu que vários fatores endógenos podem influenciar a cor da madeira, como características anatômicas, camadas de crescimento, e o tamanho e tipo de parênquima. Já Mady (2000) comenta que a cor é derivada, principalmente, da composição química de substâncias presentes no xilema (polifenóis, flavonóides, estilbenos, quinonas, entre outras). Segundo Burtin et al., 
(1998), a cor também é alterada pela umidade, temperatura e a degradação advindas de reações fotoquímicas dos componentes químicos presentes em sua estrutura.

A cor, dentre as propriedades organolépticas, é a característica que apresenta maior subjetividade, por estar relacionada diretamente com a sensibilidade psicofísica do observador (ZENID; CECCANTINI, 2007). A busca pela quantificação deste parâmetro pode levar a redundâncias com base em métodos de colorimetria pouco eficazes, diminuindo sua precisão, como no caso da Carta de Munsell (Munsel Soil Color Chart - Munsell), uma metodologia desenvolvida para a classificação colorimétrica de solos.

Outra opção é, o sistema CIE $L^{*} a^{*} b^{\star}$ de 1976 (Comission International de L'Eclairage ou Comissão Internacional de Iluminantes) que define a cor quantitativamente por meio dos parâmetros de luminosidade $\left(\mathrm{L}^{\star}\right)$, coordenada cromática verde-vermelho $\left(\mathrm{a}^{\star}\right)$, coordenada cromática azul-amarelo $\left(b^{\star}\right)$, saturação da cor $(C)$ e ângulo de tinta $\left(h^{\star}\right)$ (CAMARGO; GONÇALEZ, 2001).

Diante do exposto o objetivo deste trabalho foi caracterizar quantitativamente a cor da madeira de trinta espécies tropicais, propondo classes de agrupamento pela similaridade dos parâmetros colorimétricos e massa específica.

\section{Material e Métodos}

Foram selecionadas madeiras (cerne) de trinta espécies comercializadas no país (Astronium lecointei Ducke - Anacardiaceae; Aspidosperma polyneuron Mueller - Apocynaceae; Handroanthus albus (Chamiso) Mattos, Tabebuia sp. Bignoniaceae; Ochroma pyramidale Urban. - Bombacaceae; Trattinickia buserifolia Mart. -Burseraceae; Caryocar villosum (Aubl.) Pers. - Caryocaraceae; Goupia glabra Aubl. - Celastraceae; Ferreirea spectabilis Hassl, Dypteryx odorata (Aubl.) Willd., Caesalpinia echinata Lam. Dialium guianense
(Aubl.) Sandwith., Schizolobium amazonicum Huber, Hymeneae courbaril Linneaeus., Apuleia leiocarpa (Vogel) Macbr., Hymenolobium petraeum Ducke., Cedrelinga catenaeformis Ducke. - Fabaceae; Ocotea sp., Mezilaurus itauba (Meisn.) Taub. - Lauraceae; Bertholletia excelsa Humb e Bonpl. - Lecytidadeae; Swietenia macrophylla King, Cedrella Fissilis Vellozo - Meliaceae; Clarisia racemosa Ruiz e Pav., Bagassa guianensis Aubl., Morus sp. - Moraceae; Micropholis venulosa (Mart e Eichler) Pierre - Sapotaceae; Simarouba amara Aubl. - Simaroubaceae; Tectona grandis Linn. - Lamiaceae; Qualea sp., Erisma uncinatum Warm. - Vochysiaceae). Para cada espécie foram retiradas 5 amostras prismáticas com as dimensões de $6 \mathrm{~cm} \times 1 \mathrm{~cm} \times 13 \mathrm{~cm}$, provenientes da xiloteca da Universidade Federal de Mato Grosso, campus Sinop-MT. Devido a oxidação da superfície da madeira que altera sua tonalidade natural, as amostras foram submetidas à lixamento com lixa de granulometria de 200 , bem como ao acondicionamento em sala climatizada a $12 \%$ de umidade.

A caracterização colorimétrica foi realizada no plano tangencial da madeira por meio de um colorímetro com resolução de 3nm de iluminação difusa, iluminante D65 composto por lâmpada de xenônio com ângulo de observação $10^{\circ} \mathrm{e}$ área de iluminação de $11 \mathrm{~mm}$ de diâmetro. Um total de 3 leituras foram feitas por amostra, obtendo-se os parâmetros colorimétricos $L^{\star}, a^{*}, b^{\star}, C^{*} e h$.

A massa específica aparente a $12 \%$ de umidade, foi obtida com base na NBR 7190 (ASSOCIAÇÃO BRASILEIRA DE NORMAS TÉCNICAS - ABNT, 1997), pela razão entre massa e volume. A massa foi obtida por pesagem em balança analítica de precisão (resolução de 0,001 g). Já o volume foi determinado pela mensuração das dimensões nas direções longitudinal, radial e tangencial, com auxílio de um paquímetro de resolução de $0,01 \mathrm{~mm}$. 
Os dados foram analisados por meio de análise de variância (ANOVA) e testes de Scott-Knott, em nível de significância de $5 \%$ de probabilidade de erro, seguido de agrupamento pelo grau de similaridade pela análise multivariada de Cluster, utilizando-se a distância euclidiana quadrática, o método de ligação entre grupos (Between-groups linkage) e estandardização pelo número de pontuações $Z$, com segmentação dos grupos definida pela linha de Fenon, traçada no dendrograma em razão da distância euclidiana.

\section{Resultados e Discussão}

As madeiras apresentaram um amplo espectro de variação dos parâmetros colorimétricos básicos: $\mathrm{a}^{\star}$ variou a intensidade de vermelho de 3,03 a 18,$98 ; b^{*}$ variou a intensidade do amarelo entre 4,95 a 32,$90 ; L^{\star}$ variou o escurecimento entre 40,42 a 83,60. Em relação ao $h^{\star}$, verificou-se uma variação entre 14,43 a 77,18 , indicando uma maior intensidade de tons encontrados na coordenada $\mathrm{a}^{*}$, até 82,30 indicando maior intensidade de tons presentes na coordenada $b^{\star}$. Por sua vez, C que é o desvio da coordenada $L^{*}$, ou seja, quanto maior o valor mais livre de branco, o parâmetro apresentou variação entre 15,55 a 33,76 (Tabela 1$)$

Dentre as espécies analisadas, D. guianenses e C. echinata apresentaram as superfícies mais escurecidas, o que pode ser verificado pelos menores valores de $\mathrm{L}^{\star} \mathrm{e}$ maiores valores de $\mathrm{a}^{\star}$. Por outo lado, as madeiras de S. amara, O. pyramidale e $S$. amazonicum destacam-se como sendo as mais claras na análise colorimétrica por possuir maiores médias de $\mathrm{L}^{\star}$ e baixos valores de $\mathrm{a}^{*}$.

Madeiras como Morus sp., C. racemosa e A. leiocarpa apresentaram os maiores valores de $\mathrm{b}^{*}$, considerando a pureza da cor, observa-se que a primeira madeira é a mais puras dentre as espécies aqui analisadas confirmado pelo grau de angulação de tinta.
Para as madeiras de S. amara, $H$. courbaril, D. odorata e T. grandis verificou-se que os valores médios de $\mathrm{L}^{*}, \mathrm{a}^{\star}, \mathrm{b}^{\star}, \mathrm{C}$ $\mathrm{e} \mathrm{h}^{\star}$ foram maiores que os descritos por Costa et al. (2011a,b), Paula et al. (2016), Garcia et al. (2016) e Stangerlin et al. (2013). Entretanto, ressalta-se que as diferenças entre os valores absolutos verificados neste estudo em comparação aos descritos na literatura estão relacionados a fatores como, idade, teor de umidade, características anatômicas e químicas.

A massa específica aparente a $12 \%$ de umidade variou entre $0,250 \mathrm{~g} / \mathrm{cm}^{3}$ e $1,040 \mathrm{~g} / \mathrm{cm}^{3}$, em que as espécies de O. pyramidale e C. echinata apresentaram o menor e o maior valor respectivamente. Os valores de massa específica aparente encontrados neste trabalho para C. echinat e O. pyramidale estão dentro da variação encontrados por Longui (2005) e Lobão et al., (2008).

Comparando as espécies por análise multivariada de Cluster com base na segmentação dos grupos obtida por meio de uma distância de corte igual a 3, a qual foi escolhida visualmente no dendrograma em razão da distância euclidiana, observa-se que as espécies foram separadas em 5 grupos (Tabela 2).

Pode-se inferir que o grupo I das espécies D. guianense e C. echinata apresenta-se como o mais distinto, o que pode ser explicado por estas espécies possuírem alta massa específica, os maiores valores de $\mathrm{L}^{\star} \mathrm{e} \mathrm{a}^{\star}$, e os menores em $\mathrm{h}^{\star} \mathrm{e} \mathrm{b}^{\star}$. O grupo II é o grupo de espécies que apresentam os menores valores de $L^{\star}$ e de massa específica, nele representado pelas espécies S. amazonicum, O. pyramidale e S. amara.

A madeira de Morus sp. agrupou distintamente das demais o que pode ser relacionado a elevada intensidade do parâmetro $b^{\star}$ também indicada pelo teste de Scott-Knott, o qual diferenciou das demais espécies.

O Grupo III, é o das madeiras de tonalidade marrom escura e alta massa específica, com coloração menos pura e com 
tendências de tom amarelado. O Grupo IV está separado em dois subgrupos o primeiro composto por E. uncinatum, $S$. macrophylla, H. courbaril, A. lecointei, A. polyneuron, e Qualea sp. espécies de massa específica moderadamente alta $\left(0,626-0,962 \mathrm{~g} / \mathrm{cm}^{3}\right), \mathrm{a}^{\star}$ entre 12 e $15,6, \mathrm{~h}^{\star}$ maior que 55 e menor que 60 , com $C$ entre 21 e 28,6; já o segundo subgrupo composto por C. catenaeformis e $M$. venulosa diferindo das espécies do primeiro subgrupo por apresentarem menores valores de massa específica e $\mathrm{C}$, e maiores valores de $\mathrm{L}^{*}$.

Grupo V é dividido em 3 subgrupos, o primeiro com as

Tabela 1. Parâmetros colorimétricos e massa específica aparente da madeira das 30 espécies tropicais.

Table 1. Colorimetric parameters and density of wood from 30 tropical species.

\begin{tabular}{|c|c|c|c|c|c|c|}
\hline Espécie & $\mathbf{L}^{*}$ & $a^{*}$ & $\mathbf{b}^{*}$ & $\mathrm{C}$ & $\mathbf{h}^{*}$ & $\mathrm{ME} \mathrm{g} / \mathrm{cm}^{3}$ \\
\hline A. lecointei & $53,61( \pm 2,81) \mathrm{D}$ & $14,33( \pm 1,11) \mathrm{I}$ & $23,76( \pm 1,34) \mathrm{G}$ & $27,75( \pm 1,65) \mathrm{F}$ & $58,93( \pm 1,13) \mathrm{C}$ & $0,87( \pm 0,11) \mathrm{E}$ \\
\hline A. leiocarpa & $63,92( \pm 2,61) \mathrm{G}$ & $11,46( \pm 1,22) \mathrm{G}$ & $28,74( \pm 1,59) \mathrm{I}$ & $33,12( \pm 7,8) \mathrm{H}$ & $68,19( \pm 2,9) \mathrm{E}$ & $0,84( \pm 0,02) \mathrm{E}$ \\
\hline A. polyneuron & $55,32( \pm 1,03) \mathrm{E}$ & $15,37( \pm 1,57) \mathrm{I}$ & $22,04( \pm 1,59) \mathrm{E}$ & $26,88( \pm 2,01) \mathrm{F}$ & $55,15( \pm 2,06) \mathrm{C}$ & $0,87( \pm 0,05) \mathrm{E}$ \\
\hline B. excelsa & $59,52( \pm 2,19) \mathrm{F}$ & $11,49( \pm 0,76) \mathrm{G}$ & $22,36( \pm 0,92) \mathrm{E}$ & $25,14( \pm 1,12) \mathrm{E}$ & $62,83( \pm 0,93) \mathrm{D}$ & $0,72( \pm 0,06) \mathrm{D}$ \\
\hline B. guianensis & $56,05( \pm 2,03) \mathrm{E}$ & $10,87( \pm 0,87) \mathrm{G}$ & $25,93( \pm 1,83) \mathrm{H}$ & $28,12( \pm 1,91) \mathrm{E}$ & $67,25( \pm 1,39) \mathrm{E}$ & $0,84( \pm 0,03) \mathrm{E}$ \\
\hline C. catenaeformis & $56,56( \pm 3,62) \mathrm{E}$ & $10,11( \pm 1,06) \mathrm{F}$ & $17,79( \pm 0,88) \mathrm{C}$ & $20,48( \pm 1,08) \mathrm{C}$ & $60,43( \pm 2,45) \mathrm{D}$ & $0,61( \pm 0,08) \mathrm{C}$ \\
\hline C. echinata & $42,62( \pm 2,16) \mathrm{A}$ & $18,96( \pm 1,68) \mathrm{J}$ & $16,36( \pm 1,99) \mathrm{B}$ & $25,08( \pm 2,26) \mathrm{E}$ & $40,74( \pm 2,9) \mathrm{B}$ & $1,04( \pm 0,01) \mathrm{G}$ \\
\hline C. fissilis & $62,86( \pm 2,37) \mathrm{H}$ & $12,72( \pm 0,27) \mathrm{H}$ & $23,69( \pm 0,41) \mathrm{G}$ & $26,89( \pm 0,34) \mathrm{F}$ & $61,77( \pm 0,76) \mathrm{D}$ & $0,43( \pm 0,15) \mathrm{B}$ \\
\hline C. racemosa & $58,67( \pm 1,63) \mathrm{F}$ & $9,78( \pm 1,23) \mathrm{F}$ & $29,64( \pm 2,26) \mathrm{I}$ & $29,94( \pm 1,84) \mathrm{G}$ & $68,78( \pm 3,32) \mathrm{E}$ & $0,82( \pm 0,07) \mathrm{E}$ \\
\hline C. villosum & $66,87( \pm 3,17) \mathrm{H}$ & $9,21( \pm 1,42) \mathrm{E}$ & $24,36( \pm 2,81) \mathrm{G}$ & $26,06( \pm 2,98) \mathrm{E}$ & $69,29( \pm 2,27) \mathrm{E}$ & $0,84( \pm 0,02) \mathrm{E}$ \\
\hline D. guianense & $40,42( \pm 1,99) \mathrm{A}$ & $18,98( \pm 1,57) \mathrm{J}$ & $4,95( \pm 1,68) \mathrm{A}$ & $19,66( \pm 1,85) \mathrm{C}$ & $14,43( \pm 3,85) \mathrm{A}$ & $0,81( \pm 0,04) \mathrm{E}$ \\
\hline D. odorata & $61,27( \pm 3,74) \mathrm{G}$ & $11,11( \pm 2,05) \mathrm{G}$ & $25,64( \pm 1,35) \mathrm{H}$ & $28,03( \pm 1,18) \mathrm{F}$ & $66,89( \pm 4,44) \mathrm{E}$ & $0,91( \pm 0,12) \mathrm{E}$ \\
\hline E. uncinatum & $48,35( \pm 1,09) \mathrm{C}$ & $12,26( \pm 1,13) \mathrm{H}$ & $17,83( \pm 0,96) \mathrm{C}$ & $21,64( \pm 1,37) \mathrm{D}$ & $55,61( \pm 1,41) \mathrm{C}$ & $0,63( \pm 0,04) \mathrm{C}$ \\
\hline F. spectabilis & $58,22( \pm 2,9) \mathrm{F}$ & $9,89( \pm 1,52) \mathrm{F}$ & $25,07( \pm 1,91) \mathrm{H}$ & $27( \pm 1,77) \mathrm{F}$ & $68,41( \pm 3,56) \mathrm{E}$ & $0,81( \pm 0,1) \mathrm{E}$ \\
\hline G. glabra & $55,66( \pm 2,94) \mathrm{E}$ & $14,78( \pm 1,92) \mathrm{I}$ & $23,35( \pm 3,01) \mathrm{F}$ & $27,68( \pm 3,16) \mathrm{F}$ & $57,58( \pm 3,46) \mathrm{C}$ & $0,87( \pm 0,06) \mathrm{E}$ \\
\hline H. albus & $45,26( \pm 3,22) \mathrm{B}$ & $7,98( \pm 2,77) \mathrm{D}$ & $15,72( \pm 3,53) \mathrm{B}$ & $17,67( \pm 4,32) \mathrm{B}$ & $63,5( \pm 3,82) \mathrm{D}$ & $1,07( \pm 0,1) \mathrm{G}$ \\
\hline H. courbaril & $52,1( \pm 4,08) \mathrm{D}$ & $15,64( \pm 1,32) \mathrm{I}$ & $24,33( \pm 3,29) \mathrm{G}$ & $28,99( \pm 2,96) \mathrm{F}$ & $57,02( \pm 3,94) \mathrm{C}$ & $0,96( \pm 0,05) \mathrm{F}$ \\
\hline H. petraeum & $62,98( \pm 3,76) \mathrm{H}$ & $12,04( \pm 1) \mathrm{H}$ & $23,45( \pm 0,92) \mathrm{F}$ & $26,38( \pm 0,69) \mathrm{E}$ & $62,8( \pm 2,55) \mathrm{D}$ & $0,66( \pm 0,04) \mathrm{D}$ \\
\hline M. venulosa & $60,82( \pm 2,76) \mathrm{G}$ & $10,85( \pm 0,91) \mathrm{G}$ & $21,14( \pm 0,86) \mathrm{E}$ & $22,15( \pm 0,87) \mathrm{D}$ & $54,63( \pm 8,92) \mathrm{C}$ & $0,59( \pm 0,05) \mathrm{C}$ \\
\hline M. itauba & $53,06( \pm 2,36) \mathrm{D}$ & $5,11( \pm 1,3) \mathrm{B}$ & $22,07( \pm 1,89) \mathrm{E}$ & $22,68( \pm 1,98) \mathrm{D}$ & $76,99( \pm 3,09) \mathrm{G}$ & $0,85( \pm 0,09) \mathrm{E}$ \\
\hline Morus sp. & $64,99( \pm 2,2) \mathrm{G}$ & $7,47( \pm 1,07) \mathrm{D}$ & $32,9( \pm 1,61) \mathrm{J}$ & $33,76( \pm 1,58) \mathrm{H}$ & $77,18( \pm 1,91) \mathrm{G}$ & $0,72( \pm 0,09) \mathrm{D}$ \\
\hline O. pyramidale & $79,14( \pm 1,42) \mathrm{J}$ & $3,47( \pm 0,37) \mathrm{A}$ & $15,15( \pm 3,15) \mathrm{B}$ & $15,55( \pm 3,14) \mathrm{A}$ & $76,77( \pm 1,59) \mathrm{G}$ & $0,25( \pm 0,04) \mathrm{A}$ \\
\hline Ocotea sp. & $56,72( \pm 1,57) \mathrm{E}$ & $8,84( \pm 1,71) \mathrm{E}$ & $22,45( \pm 1,94) \mathrm{E}$ & $24,17( \pm 2,13) \mathrm{E}$ & $68,6( \pm 3,61) \mathrm{E}$ & $0,66( \pm 0,08) \mathrm{D}$ \\
\hline Qualea sp. & $56,46( \pm 4,33) \mathrm{E}$ & $14,72( \pm 1,05) \mathrm{I}$ & $24,54( \pm 0,68) \mathrm{G}$ & $28,64( \pm 0,68) \mathrm{F}$ & $59,05( \pm 2,09) \mathrm{C}$ & $0,76( \pm 0,04) \mathrm{E}$ \\
\hline S. amara & $83,6( \pm 2,14) \mathrm{K}$ & $3,03( \pm 0,39) \mathrm{A}$ & $23,31( \pm 2,89) \mathrm{F}$ & $22,86( \pm 1,76) \mathrm{D}$ & $82,3( \pm 1,24) \mathrm{H}$ & $0,41( \pm 0,01) \mathrm{B}$ \\
\hline S. amazonicum & $75,34( \pm 5,78) \mathrm{I}$ & $6,68( \pm 3,04) \mathrm{C}$ & $19,62( \pm 1,62) \mathrm{D}$ & $20,89( \pm 2,2) \mathrm{C}$ & $71,67( \pm 7,17) \mathrm{F}$ & $0,48( \pm 0,05) \mathrm{B}$ \\
\hline S. macrophylla & $48,79( \pm 5,24) \mathrm{C}$ & $14,35( \pm 1,38) \mathrm{I}$ & $22,13( \pm 2,81) \mathrm{E}$ & $25,75( \pm 3,03) \mathrm{E}$ & $56,88( \pm 2,85) \mathrm{C}$ & $0,67( \pm 0,14) \mathrm{D}$ \\
\hline T. grandis & $57,03( \pm 3,73) \mathrm{E}$ & $8,04( \pm 0,37) \mathrm{D}$ & $20,11( \pm 1,33) \mathrm{D}$ & $21,66( \pm 1,28) \mathrm{D}$ & $68,15( \pm 1,3) \mathrm{E}$ & $0,58( \pm 0,05) \mathrm{C}$ \\
\hline T. burserifolia & $69,11( \pm 9,15) \mathrm{H}$ & $10,25( \pm 4,25) \mathrm{F}$ & $24,39( \pm 2,86) \mathrm{G}$ & $26,48( \pm 4,01) \mathrm{E}$ & $67,97( \pm 7,37) \mathrm{E}$ & $0,64( \pm 0,09) \mathrm{C}$ \\
\hline Tabebuia sp. & $48,34( \pm 1,92) \mathrm{C}$ & $5,73( \pm 1,44) \mathrm{B}$ & $17,71( \pm 1,45) \mathrm{C}$ & $18,68( \pm 1,34) \mathrm{B}$ & $71,96( \pm 4,89) \mathrm{F}$ & $1,09( \pm 0,09) \mathrm{G}$ \\
\hline$C V \%$ & 5,79 & 14,85 & 9,08 & $11,35 \%$ & 7,81 & 9,74 \\
\hline
\end{tabular}

Médias seguidas de mesma letra não diferem estatisticamente pelo teste de Scott-Knott em 5\% de significância de probabilidade de erro. 
Tabela 2. Agrupamento das espécies por análise multivariada de Cluster segundo seus parâmetros colorimétricos e massa específica aparente e nomenclatura da cor.

Table 2. Grouping of species by Multivariate Clustering Analysis according to their colorimetric parameters and density and color name.

\begin{tabular}{|c|c|c|c|c|c|}
\hline $\begin{array}{c}\text { Grupos de simila- } \\
\text { ridade }\end{array}$ & Espécie & Nome da cor $^{1}$ & $\begin{array}{l}\text { Grupos de si- } \\
\text { milaridade }\end{array}$ & Espécie & Nome da cor ${ }^{1}$ \\
\hline \multirow{2}{*}{ Grupo I } & D. guianense & Roxo & \multirow{13}{*}{ Grupo V } & B. guianensis & Marrom-oliva \\
\hline & C. echinata & Vermelho & & Qualea sp. & Amarelo-amarronzado \\
\hline \multirow{3}{*}{ Grupo II } & S. amazonicum & Cinza-rosado & & Ocotea sp. & Oliva-amarelado \\
\hline & O. pyramidale & $\mathrm{NC}^{2}$ & & T. grandis & Oliva-amarelado \\
\hline & S. amara & Branco-acinzentado & & F. spectabilis & Marrom-oliva \\
\hline & H. albus & Oliva & & C. racemosa & Marrom-oliva \\
\hline Grupo III & Tabebuia sp. & Oliva & & B. excelsa & Marrom-oliva \\
\hline & M. itauba & Oliva-amarelado & & D. odorata & Marrom-escuro \\
\hline \multirow{8}{*}{ Grupo IV } & E. uncinatum & Marrom escuro & & C. fissilis & Rosa \\
\hline & S. macrophylla & Amarelo-amarronzado & & H. petraeum & Rosa \\
\hline & H. courbaril & Marrom-avermelhado & & A. leiocarpa & Marrom-oliva \\
\hline & A. lecointei & Amarelo-amarronzado & & C. villosum & Marrom-oliva \\
\hline & A. polyneuron & Marrom-avermelhado & & T. burserifolia & Marrom-oliva \\
\hline & G. glabra & Amarelo-amarronzado & \multirow[t]{3}{*}{$\mathrm{NC}$} & \multirow[t]{3}{*}{ Morus sp. } & \multirow[t]{3}{*}{$\mathrm{NC}$} \\
\hline & M. venulosa & Marrom-claro & & & \\
\hline & C. catenaeformis & Marrom-claro & & & \\
\hline
\end{tabular}

${ }^{1}$ Nome da cor segundo intervalos cromáticos de Camargo e Gonçalez (2001); 2 Não classificado (NC).

espécies T. grandis e Ocotea sp. que dentro do grupo apresentou os menores valores de $\mathrm{L}^{\star}, \mathrm{C}$ e $\mathrm{a}^{*}$; outro subgrupo é o das espécies C. vilosum e T. buserifolia que dentro do grupo diferencia-se dos demais por apresentarem maiores valores de saturação de branco; o ultimo subgrupo composto por C. fissilis, H. petraeum, B. excelsa, B. guianensis, F. espectabilis, D. odorata, C. racemosa e A. leiocarpa que diferenciou dos demais por apresentar valores de saturação por branco entre 58,221 a 63,917 .Quando comparados os valores obtidos por este trabalho com os intervalos cromáticos propostos por Camargo e Gonçalez (2001) pode-se observar que o Grupo I é composto por madeiras de tonalidades roxo e vermelho; Grupo II, de madeiras branco-acinzentadas e cinza-rosadas; Grupo III, madeiras de tonalidades Oliva a Oliva-amarelada, Grupo IV, madeiras amarelo-amarronzado, marrom-claro, marromavermelhado a um marrom escuro; Grupo V, por sua maioria de madeiras de tonalidades marrom-oliva, oliva-amarelado, amarelo-amarelado e rosa. Apesar da abrangência das classes cromáticas propostas pelos autores, as espécies O. pyramidale e Morus sp. não foram passiveis de classificação.

\section{Conclusões}

- As trinta madeiras analisadas foram agrupadas em cinco grupos sendo o Grupo I com 6,67\%, Grupo II e III com 10\% cada, Grupo IV com 30\% e o Grupo V com 40\% das espécies, sendo que a espécie de Morus spp. foi a única espécie a não se agrupar devida às suas características colorimétricas.

- A classificação de cluster proporcionou a segregação de madeiras formando grupos heterogêneos, com base na diversidade colorimétrica e massa específica, em que madeiras mais escuras e densas foram classificadas em grupos distintos daquelas mais claras e leves. 
- Recomenda-se para futuros estudos a inclusão de parâmetros químicos e anatômicos para que auxiliem na melhor interpretação dos agrupamentos.

\section{Referências}

BURTIN, P.; JAY-ALLEMAND, C.; CHARPENTIER, J. P.; JANIN, G. Natural wood colouring process in Juglans sp. (J. nigra, J. regia and hybrid J. nigra $23 \times \mathrm{x}$. regia) depends on native phenolic compounds accumulated in the transition zone between sapwood and heartwood. Trees, Berlin, v. 12, n. 5 , p. $258-264,1998$.

CAMARGOS, J. A. A.; GONÇALEZ, J. C.; A colorimetria aplicada como instrumento na elaboração de uma tabela de cores de madeira. Brasil Floresta, n.71, 2001.

COSTA, J. de A.; GONÇALEZ, J. C.; CAMARGO, J. A. A.; GOMES, I. A. S.; Fotodegradação de duas espécies de madeiras tropicais: jatobá (Hymenaea courbaril) e tauari (Couratari oblongifolia) submetidas à radiação ultravioleta, Cerne, Lavras, v. 17, n. 1, p. 133-139, 2011.

COSTA, M. de A.; COSTA, A. F. da; PASTORE, T. C. M.; BRAGA, J. W. B.; GONÇALEZ, J. C.; Caracterização do ataque de fungos apodrecedores de madeiras através da colorimetria e da espectroscopia de infravermelho, Ciência Florestal, Santa Maria, v. 21, n. 3, p. 567-577, 2011.

DONI, M. V., Análise de Cluster: métodos hierárquicos e de particionamento, São Paulo: Universidade Presbiteriana Mackenzie, São Paulo, 2004.

GARCIA, R. A.; MARNONIO, G. B.; Variação da cor da madeira de teca em função da massa específica e do teor de extrativos, Floresta e Ambiente, v.23, n. 1, p.124-134, 2016.

GONÇALEZ, J. C. Caracterisation technologique de quatre especes peu connues de la forêt Amazonienne: anatomie, chimie, couleur, propriétés physiques et mécaniques. Nancy, 1993. $445 \mathrm{f}$. Thèse (Doctorat en Sciences Forestières) - Ecole Nationale du Génie Rural, des Eaux et des Forêts, Nancy, France, 1993.

LOBÃO, M. S.; SOARES, P. R. C.; CHAGAS, M. P.; ROSERO, J. A.; SERVOLO, H.; PALMEIRAS, L. P. S. Agrupamento de espécies madeireiras por similaridade de suas características físicas e anatômicas. $5^{\circ}$ Simpósio de Pós-Graduação em Ciências Florestais, Brasilia, p. 645-659, 2008.
LONGHI, E. L. Pau-brasil e outras espécies nativas empregadas na confecção de arcos para instrumentos de corda: um estudo comparativo. 2005. 75f. Dissertação (Mestrado em Botânica) Universidade de São Paulo, Instituto de Botânica, São Paulo, 2005.

MADY, F. T. M. Conhecendo a madeira: informações sobre 90 espécies comerciais. Manaus: SEBRAE/AM/Programa de Desenvolvimento Empresarial e Tecnológico, 2000. 212 p.

MARTINS, M. da F., BELTRAME, R., DELUCIS, R. de A., GATTO, R. de A., CADEMARTORI, D. A., P. H. G. de, dos SANTOS G. A. Colorimetria como ferramenta de agrupamento de madeira de clones de eucalipto, Pesquisa Florestal Brasileira, Colombo, v. 35, n.84, p. 443-449, 2015.

MORI, C. L. S. DE O.; MORI, F. A.; LIMA, J. T.; TRUGILHO, P. F.; OLIVEIRA, A. C.; Influência das características tecnológicas na cor da madeira de eucaliptos, Ciência Florestal, Santa Maria, v. 14, n. 2, p. 123-132, 2004.

NISHINO, Y.; JANIN, G.; YAMUDA, Y.; KITANO, D. Relations between colorimetric values and densities of sapwood. Journal of Wood Science, v. 46, n. 4, p. 267-272, 2000.

DE PAULA, M. H.; MESQUITA, R. R. S. de; GONÇALEZ, J. C.; RIBEIRO, E. S.; SOUZA, R. S.; Utilização de métodos não destrutivos para caracterização simplificada da madeira de cumaru (Dipteryx odorata Willd), Biodiversidade, v.15, n.2, p.136-149, 2016.

SILVA, E. dos S.; STANGELIN, D. M.; GATTO, D. A.; CALEGARI, L.; PARIS, E.; Colorimetria da madeira de oito espécies nativas do estado do Rio Grande do Sul, Brasil, Ciência da Madeira, v. 6, n. 1, p. 31-37, 2015.

STANGELIN, D. M.; COSTA, A. F. da; GARLET, A.; PASTORE, T. C. M.; Resistência natural da madeira de três espécies amazônicas submetidas ao ataque de fungos apodrecedores. Ciência da Madeira, Pelotas, v.4, n.1, p. 15-32, 2013.

ZENID, G. J.; E CECCANTINI, G. C. T.; IPT -Identificação macroscópica de madeiras, setembro de 2007. 